\title{
Metastatic Crohn's disease of thigh and forearm
}

\author{
J H TWEEDIE AND B G McCANN \\ From the West Norwich Hospital, Norwich, Norfolk
}

SUmmary Cutaneous involvement with Crohn's disease at a site separated from the gastrointestinal tract by normal skin is termed metastatic Crohn's disease. ${ }^{1}$ Such a complication is extremely rare and previous reports have emphasised the flexural distribution of the ulcerated lesions. We report a patient who developed Crohn's disease involving the skin of the thigh and forearm several months after a panproctocolectomy for colorectal Crohn's disease.

\section{Case report}

A 65 year-old man presented with a 10 day history of passing watery stools every three to four hours. Two days before admission he noticed blood in the stool and experienced abdominal pain on defaecation. Four years previously he had had a resection of an abdominal aortic aneurysm.

Examination showed a fit man, with blood stained fluid faeces noted on rectal examination. Sigmoidoscopy disclosed friable granular mucosa with blood and mucopus in the lumen of the bowel. Rectal biopsy specimens showed moderate acute inflammation with focal crypt abscess formation but no granulomata and no evidence of ischaemic changes in the rectum.

Other investigations showed normal haemoglobin concentration of $14.8 \mathrm{~g} / \mathrm{dl}$, mild leucocytosis, $14 \cdot 2 \times 10^{9}$, and a raised erythrocyte sedimentation rate $(45 \mathrm{~mm}$ in the first hour). Apart from a raised alkaline phosphatase (117 IU/l), liver function tests and the blood concentration of urea and other electrolytes were normal. Chest radiography showed no evidence of sarcoidosis or tuberculosis. Sputum, urine, and stool cultures failed to show any pathogens. Barium enema showed a total colitis with widespread deep ulceration, but the terminal ileum looked normal. Despite systemic steroids (prednisolone $10 \mathrm{mg}$ thrice daily) and salazopyrin $500 \mathrm{mg}$ four times daily his condition deteriorated and he underwent a panproctocolectomy with formation of ileostomy five weeks after pre-

Address for correspondence: Mr J H Tweedie, FRCS, West Norwich Hospital Bowthorpe Road, Norwich, Norfolk NR2 3TU.

Received for publication 30 March 1983 sentation. At operation the whole of the colon and rectum was inflamed with extensive serpiginous ulceration. The small bowel appeared normal. Histology of the resected specimen showed severe acute on chronic colitis with fissuring ulcers extending deeply into the submucosa which was very oedematous. The mucosa between the ulcers was virtually normal. There was only slight focal lymphocytic infiltration of the serosa and granulomata were not present in the bowel or the attached lymph nodes. Overall, the changes were suggestive, but not typical, of Crohn's disease of the colon and rectum.

Six months after his panproctocolectomy granulomatous lesions were apparent on his left forearm, left thigh, right buttock, and anterior abdominal

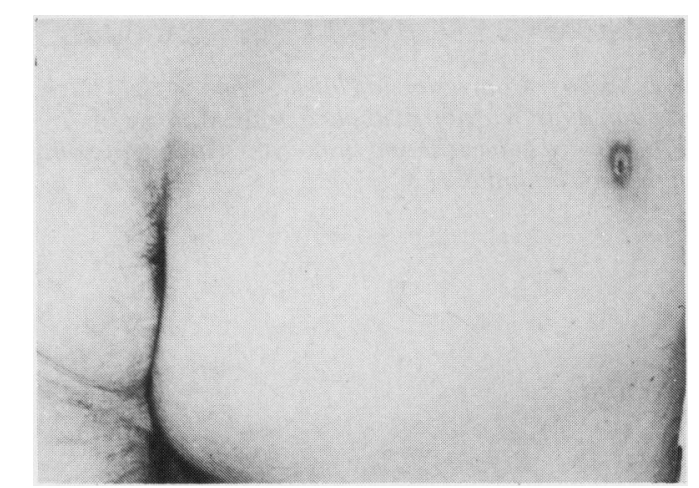

Fig. 1 Ulcer in gluteal region of right thigh. This was shallow and did not communicate with perineum. Histology of biopsy material showed the features illustrated in Fig. 2. 
wall. The lesions were raised, red, ulcerated, and had a dusky cyanosis at their periphery (Fig. 1). There was no communication between the lesions on his thigh and buttock and the perineum. There was no associated lymphadenopathy. Biopsy of the lesions revealed sarcoid like granulomas with no acid fast bacilli confirming the diagnosis of Crohn's disease (Fig. 2). He was started on prednisolone 10 $\mathrm{mg}$ thrice daily with partial healing of the ulcers. $\mathrm{He}$ remains in remission on $5 \mathrm{mg}$ thrice daily.

\section{Conclusions}

The cutaneous manifestations of Crohn's disease have been well reviewed recently. ${ }^{2}$ One group includes a wide variety of dermatoses with variable histology, including erythema nodosum, erythema multiforme, pyoderma gangrenosum, palmar erythema, rosacea, epidermolysis bullosa acquisita, cutaneous polyarteritis nodosa and the skin manifestations of malnutrition, malabsorption, and therapy. In the other group the involved skin shows certain characteristic histological features of Crohn's

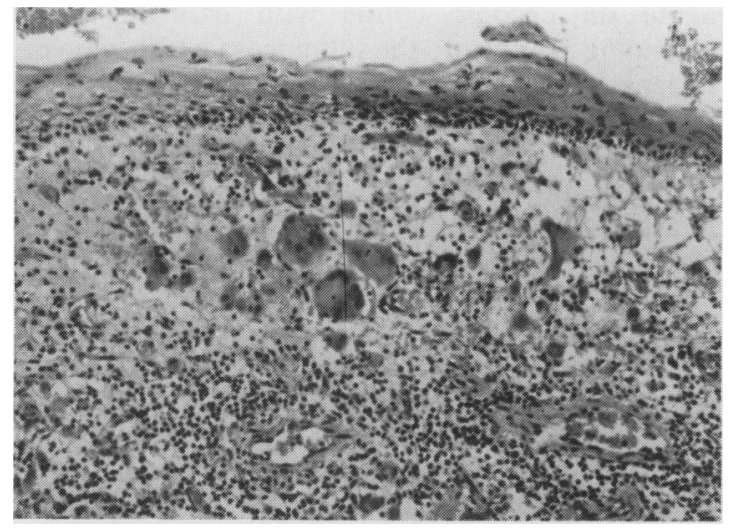

Fig. 2 Biopsy from lesion in gluteal region of right thigh. Granulomatous reaction in upper dermis. Clusters of multinucleated giant cells and histiocytes with surrounding lymphocytic infiltrate. $H \& E \times 125$. disease. ${ }^{1}$ The skin is usually involved by direct extension from the gastroiniestinal tract - for example, by perianal ulceration, fistulation, or in relation to the scar after surgery. Rarely does cutaneous Crohn's disease occur in non-contiguous skin, and in previous reports the skin involved has been in areas of skin apposition within flexures (submammary, ${ }^{1}$ retroauricular, ${ }^{3}$ anterior abdominal wall skin fold, ${ }^{4}$ and umbilicus ${ }^{5}$ ).

Ulcerated metastatic Crohn's disease of the arm and thigh has not been reported and differs from previous reports in that these are not areas of skin apposition. The absence of granulomata in the colectomy specimen and their prominence in the metastatic lesions is unusual but not unique. As in previous reports the metastatic Crohn's disease did not precede the onset of gastrointestinal disease, there was no associated lymphadenopathy, and the lesions responded to systemic steroid treatment. The aetiology of the condition remains obscure.

We thank Dr Basil Morson for kindly reviewing and reporting on the histopathology in this case and $\mathrm{Mr}$ A Cameron and $\mathrm{Mr} \mathrm{J}$ Colin for permission to publish the case admitted under their care.

\section{References}

1 Parks AG, Morson BC, Pegum JS. Crohn's disease with cutaneous involvement. Proc Roy Soc Med 1965; 58: 241 .

2 Burgdorf W. Cutaneous manifestations of Crohn's disease. J Am Acad Dermatol 1981; 5: 689-95.

3 McCallum DI, Kinmont PDC. Dermatological manifestations of Crohn's disease. Br J Dermatol 1968; 80: 1 .

4 Mountain JC. Cutaneous ulceration in Crohn's disease. Gut 1970; 11: 18.

5 Phillips RKS, Glazer G. Metastatic Crohn's disease of the umbilicus. $\mathrm{Br}$ Med $J$ 1981; 283: 887. 